\title{
MRI guided electrophysiological intervention with a voltage-based electro-anatomic mapping system
}

\author{
Zion Tse ${ }^{1^{*}}$, Charles Dumoulin², Ronald Watkins ${ }^{3}$, Israel Byrd ${ }^{4}$, Jeffrey Schweitzer ${ }^{4}$, Raymond Kwong ${ }^{1}$, \\ Gregory F Michaud ${ }^{1}$, Ehud J Schmidt ${ }^{1}$
}

From 15th Annual SCMR Scientific Sessions

Orlando, FL, USA. 2-5 February 2012

\section{Background}

MRI visualizes luminal \& vessel-wall anatomy, and identifies edema \& scar tissue, contributing to improved electrophysiological (EP) ablative procedures for treatment of Ventricular Tachycardia \& Atrial Fibrillation. MRI-guided EP interventions will be performed for the foreseeable future partially in \& outside MRI, due to the need for X-ray/Ultrasound-compliant devices. Electromagnetically tracked catheter procedures, today's norm for most EP procedure phases; vascular navigation, Electro-Anatomic-Mapping (EAM, the diagnostic and therapeutic phases), can only be performed outside MRI. Separate MRI tracking is required in MRI, complicating EP procedures which require moving in \& out of the bore $[1,2]$. Continuous catheter tracking using a single system would allow registration-free EAM in \& outside MRI. The goal was developing an MR-compatible St. Jude Medical (SJM) EnSite NavX (ESN) voltage-based tracking [3]. ESN applies $5.8 / 8.0 \mathrm{kHz}$ voltage bursts between 3 pairs of electrodes on the chest, detecting a catheter's position [4], so a challenge for intra-MRI use is MR gradient ramps which interfere with ESN operation. Minimal MR Image Quality (IQ) reduction also needs to be insured, as well as $<20 \mathrm{C}$ patient-skin heating due to components in MRI.

\section{Methods}

An MR-compatible ESN (Fig.1) minimized electrode heating and IQ reduction inside the scanner room with modified ESN surface-patches, ferrites on coaxial ESN leads (Fig.1(2)) , and RF filters at the penetration panel.

'Brigham and Women's Hopsital, Harvard Medical School, Boston, MA, USA Full list of author information is available at the end of the article
Outside the scanner room, an electronic switching circuit, triggered by a sequence's gradient-ramps and radio-frequency-pulse transmission (GR\&RF), disconnected the ESN leads from the ESN receiver when GR\&RF was detected, preventing noise from reaching it (Fig.1(3)). 2 SJM MR-compatible deflectable EP catheters were used (Fig.1(4)) [5]. The ESN was tested at $1.5 \mathrm{~T}$ in heart phantoms and swine models with varying GRE, SSFP \& FSE imaging parameters and slice orientations.

\section{Results}

Fig.1(5) shows simultaneous ESN \& MR tracking of the dual ESN\&MR-tracked catheter in the phantom, verifying the lack of interference between the 2 methods. 3 EAM \& MRI imaging experiments were performed in swine, also equipped with sensors to measure temperature at the surface ESN electrodes. Fig.1(6) shows EAM during imaging where catheter tracking of 2 catheters (reference catheter in the Coronary Sinus (CS) \& mapping catheter in the Left Atrium) was performed simultaneously on the ESN , with $<5 \%$ positional error of the CS catheter relative to its position outside MRI. IQ Reduction was $<5 \%$ in both SE \& GRE, with tracking updated during $60-80 \%$ of sequence duration. TR elongation was required in $\mathrm{TR}<4 \mathrm{~ms}$ sequences. Electrode temperature rise was $<1 \mathrm{oC}$ with $4 \mathrm{Watt} / \mathrm{kg}$ SAR sequences. Surface burns did not occur.

\section{Conclusions}

An MR-compatible ESN system permits registrationfree, minimal-heating, EAM for EP procedure in \& outside MRI, with simultaneous imaging possible. 
Fig.1: Overview of MR-compatible ESN System: Inside scanner room - modified surface patches, RF ferrities \& lowpass filters. Outside scanner room - GR\&RF switching circuit \& ESN receiver

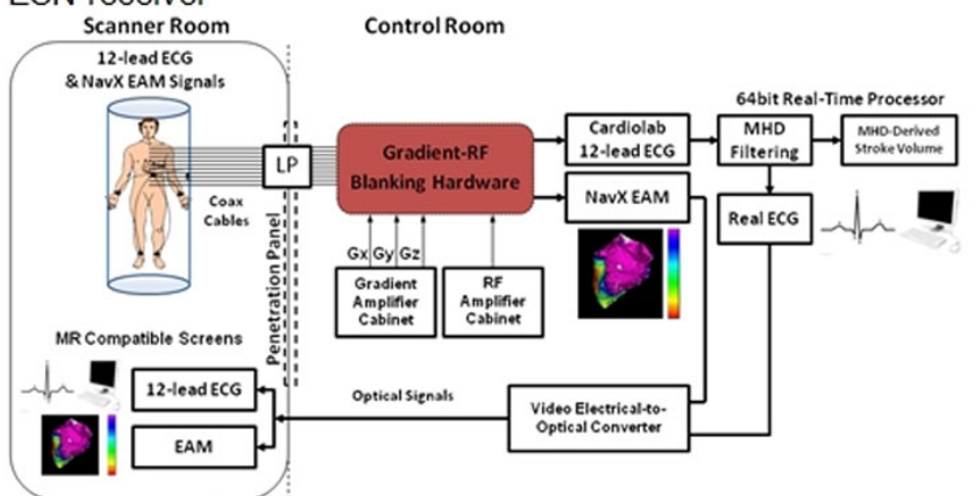

Fig.3: GR-RF blanking circuit for selective EAM signal acquisition (response time<200ns)

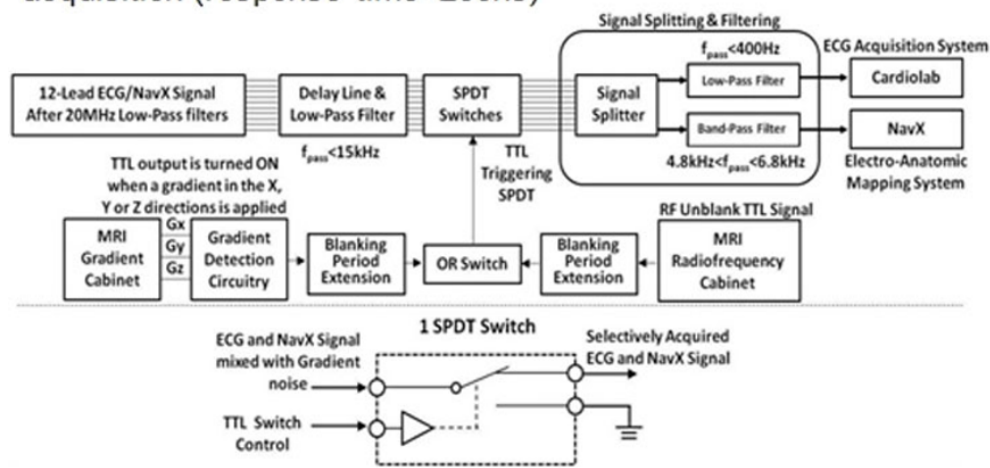

Fig.2: ESN cables with ferrite chucks implemented at RF Max

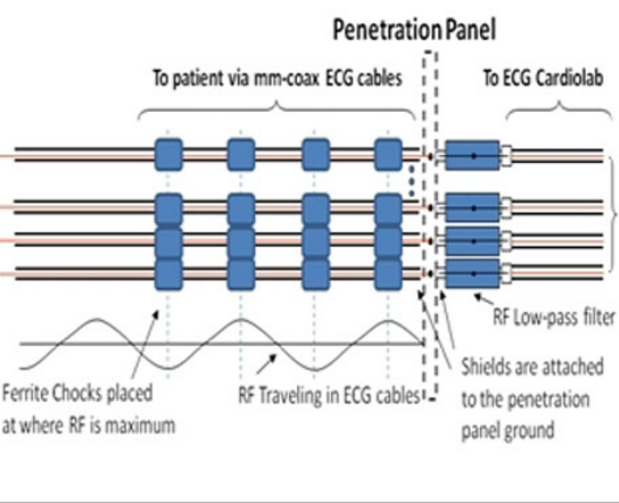

Fig.4: MR-compatible catheters (a) non-irrigated catheters with $5 \mathrm{MR}$ tracked \& 2 NavX electrodes, (b) irrigated with 8 NavX electrodes

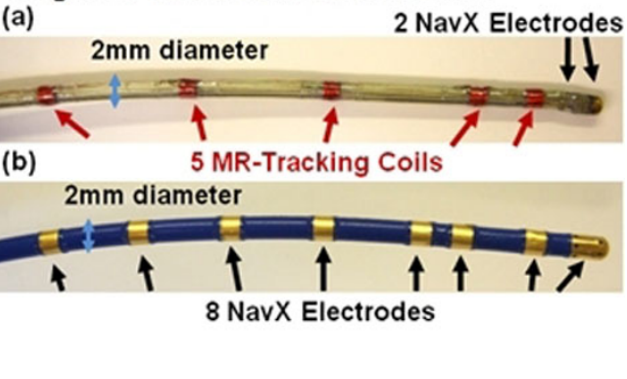

Fig.5: Simultaneous ESN\&MR tracking in a heart phantom using an MR- \& ESN- tracked catheter

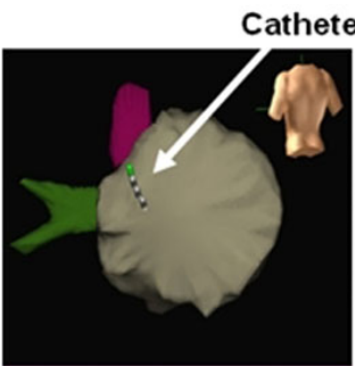

ESN EAM during Imaging

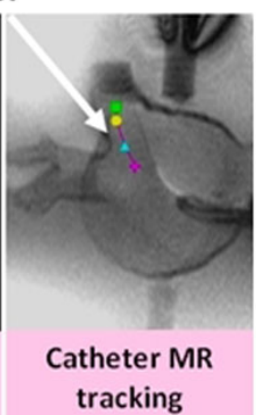

Fig.6: Navx-based EAM during swine MRI imaging. Reference catheter in Coronary Sinus and mapping catheter in Left Atrium

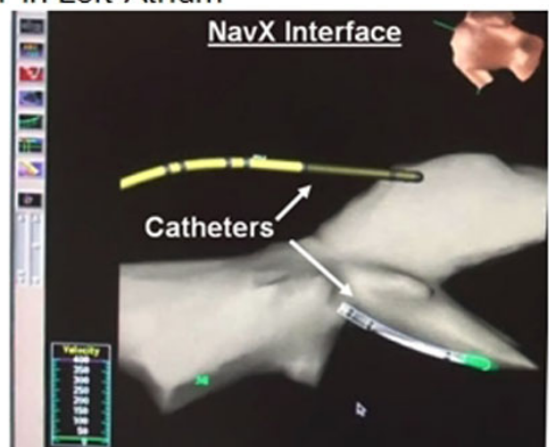

Figure 1

\section{Author details}

'Brigham and Women's Hopsital, Harvard Medical School, Boston, MA, USA 2University of Cincinnati College of Medicine, Cincinnati, OH, USA. ${ }^{3}$ Standford University, Standford, CA, USA. ${ }^{4}$ St Jude Medical, Inc., St Paul, MN, USA.

Published: 1 February 2012

References

1. Dukkipati : Circulation'08.

2. Schmidt : Circulation A\&E'09.
3. Nademanee : JACC '04..

4. Early : Eur. Heart J '06.

Tse: ISMRM \& SCMR '10\&'11.

doi:10.1186/1532-429X-14-S1-P206

Cite this article as: Tse et al:: MRI guided electrophysiological

intervention with a voltage-based electro-anatomic mapping system.

Journal of Cardiovascular Magnetic Resonance 2012 14(Suppl 1):P206. 